IOS Press

\title{
Review
}

\section{COVID-19: Significance of antibodies}

\author{
Sara Assadiasl ${ }^{\mathrm{a}}$, Yousef Fatahi ${ }^{\mathrm{b}, \mathrm{c}}$, Mahdi Zavvar $^{\mathrm{d}}$ and Mohammad Hossein Nicknam ${ }^{\mathrm{a}, \mathrm{e}, *}$ \\ ${ }^{a}$ Molecular Immunology Research Center, Tehran University of Medical Sciences, Tehran, Iran \\ ${ }^{\mathrm{b}}$ Department of Pharmaceutical Nanotechnology, Faculty of Pharmacy, Tehran University of Medical Sciences, \\ Tehran, Iran \\ ${ }^{\mathrm{c}}$ Nanotechnology Research Centre, Faculty of Pharmacy, Tehran University of Medical Sciences, Tehran, Iran \\ ${ }^{\mathrm{d}}$ Department of Medical Laboratory Sciences, School of Allied Medical Sciences, Tehran University of Medical \\ Sciences, Tehran, Iran \\ ${ }^{\mathrm{e}}$ Department of Immunology, School of Medicine, Tehran University of Medical Sciences, Tehran, Iran
}

\begin{abstract}
The newly emerged severe acute respiratory syndrome-related coronavirus-2 (SARS-CoV-2) has recently caused pandemic Coronavirus Disease-2019 (COVID-19). Considering the serious medical, economic and social consequences of this pandemic and the lack of definite medication and vaccine it is necessary to describe natural immune responses to the SARS-CoV-2 in order to exploit them for treating the patients and monitoring the general population. Moreover, detecting the most immunogenic antigens of the virus is fundamental for designing effective vaccines. Antibodies being valuable for diagnostic therapeutic and protective purposes are suitable to be addressed in this context. Herein, we have summarized the findings of serological investigations and the outcomes of neutralizing antibodies administration in COVID-19 patients.
\end{abstract}

Keywords: COVID-19, SARS-CoV-2, corona-virus, serological test, neutralizing antibodies

\section{Coronavirus}

Coronaviruses $(\mathrm{CoV})$ are enveloped positive-sense single-stranded RNA viruses that with RNA size ranging from 26 to $32 \mathrm{~kb}$ are considered to possess the largest viral RNA genome. This large RNA covered with nucleocapsid $(\mathrm{N})$ protein is held in a phospholipid bilayer and a complex of proteins including spike glycoprotein (S) hemagglutinin-esterase (HE) membrane (M) and envelope (E) which provide a crown-like shape for coronavirus [17,34] (Fig. 1). Four common coronavirus types (alpha, beta, gamma, and delta) had been recognized among them alpha and beta types are known to cause respiratory tract infections in human $[18,66]$. SARS-CoV-2 belonging to the beta coronaviruses leads

\footnotetext{
*Corresponding author: Mohammad Hossein Nicknam, Department of Immunology, School of Medicine, Tehran University of Medical Sciences, P.O. Box: 1415564477, Tehran, Iran. E-mail: mhnicknam@sina.tums.ac.ir.
}

to a respiratory, gastrointestinal and neurological disease named coronavirus disease 2019 (COVID-19) [27]. Despite strong efforts all over the world to find a definite curation or design an effective vaccine to fight SARS-CoV-2, many of them being in different phase of clinical trials, the number of infected people and death causalities are rising rapidly. Therefore, evaluation and analysis of immune responses is necessary in affected and non-affected (or slightly affected) individuals in order to find the most influential components of immune system and take the most advantage from them to support the critical patients and design the most efficient vaccines as well as epidemiologic monitoring of general population.

\section{Immune response to coronaviruses}

The innate immunity detects coronavirus through pattern recognition receptors (PRRs) such as toll-like 


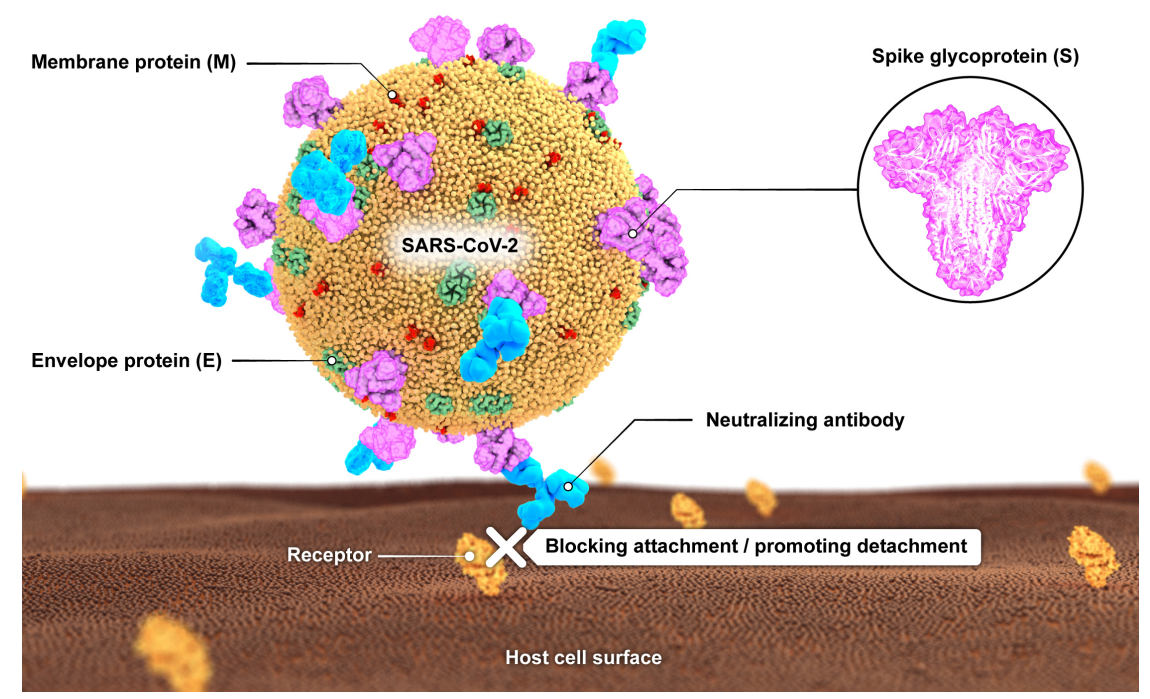

Fig. 1. A schematic illustration of SARS-CoV-2 with its main surface antigens including spike (S), membrane (M) and envelope (E) proteins. Neutralizing antibodies block virus attachment to its receptor on cell surface.

receptor (TLR); TLR-4 appears to recognize $S$ protein and to induce the production of proinflammatory cytokines through the MyD88-dependent signaling pathway. S protein mediates viral entry into the host cells by first binding to a host receptor through the receptorbinding domain (RBD) in the S1 subunit and then fusing the viral and host membranes through the S2 subunit. SARS-CoVs and MERS-CoV RBDs recognize different receptors. SARS-CoV 1 and 2 recognize angiotensin-converting enzyme 2 (ACE2), whereas MERS-CoV recognizes dipeptidyl peptidase 4 (DPP4) as their receptor. $\mathrm{S}$ protein binding to its receptor results in entrance of genomic RNA into the cytoplasm [60]. Besides, the type 2 transmembrane serine protease (TMPRSS2) in the host cell promotes viral uptake by cleaving ACE2 and activating the S protein, [25]. Once dsRNA is replicated TLR-3 could recognize it and initiate the cascades of signaling pathways to produce type I interferons (IFN) and proinflammatory cytokines. Type I IFNs improve the release of antiviral proteins in order to protect the adjacent uninfected cells. There is evidence about some accessory proteins of $\mathrm{CoV}$ (e.g. nsp1, nsp3, nsp16, ORF3b and ORF6) which interfere with TLR-3 signaling via binding to the CoV dsRNA during replication thus prevent from TLR-3 activation [46]. TLR7 and 8 are also involved in inducing immune responses to SARS-CoV-1 subsequent to the recognition of degraded viral RNA in cytoplasmic endosomes [35]. Following the activation of neutrophils and macrophages through pattern recognition receptors secretion of proinflammatory cytokines (e.g. IL-1, IL-6,
IL-8, IL-21, TNF) and presentation of viral peptides initiate; as a result, $\mathrm{T}$ cells are activated and recruited to the infection site. The dominant subtype of T lymphocytes in $\mathrm{CoV}$ infection are Th1 cells which release high amounts of IFN- $\gamma$. Cytotoxic TCD $8+$ cells play an essential role in controlling viral infections and natural killer cells participate in both killing the infected cells and IFN- $\gamma$ production [13]. On the other hand, lymphopenia has frequently been reported in severe forms of COVID-19 which was associated with the high levels of IL-6, IL-8 and TNF- $\alpha$ cytokines [14]. It is probable that viral inflammatory responses impair lymphopoiesis and/or viral particles induce lymphocyte apoptosis by direct infection [28]. The antibody response consists of producing a complex mixture of antibodies against different antigenic domains of the virus mainly $\mathrm{S}$ and $\mathrm{N}$ proteins. Antibodies defend the cells by blocking virus entrance through covering RBD of S protein and eliminating the infected cells via activation of antibody dependent cell cytotoxicity (ADCC) and complement cascade [52]. The end result of immune responses would be either disease resolution or an uncontrollable devastating inflammatory situation called cytokine storm.

\section{Diagnosis of COVID-19}

The clinical diagnosis of COVID-19 is based on symptoms of lower respiratory tract viral infections including fever, fatigue, dry cough and shortness of breath; however accumulating evidence suggests non- 
classical symptoms such as nausea, vomiting diarrhea, myalgia, anosmia and ageusia in SARS-CoV-2 infection. In severe cases complications like acute respiratory distress syndrome (ARDS), thrombosis, myocarditis, ventricular arrhythmias, hemodynamic instability cerebrovascular complications, encephalitis and liver or kidney failure may occur [66]. In addition to the general clinical symptoms and history of close contact with a diagnosed person, there are laboratory diagnostic tools to confirm COVID-19 infection. The routine laboratory tests which are used to diagnose similar infectious diseases are not applicable for COVID-19 due to the lack of specificity. For example, the white blood cell count (WBC) is usually normal in COVID-19 patients; however, as mentioned before, lymphopenia (lymphocyte count $<1000$ ) could occur in severe forms of disease. The platelet count is usually normal or mildly low but in critical cases prolonged prothrombin time, thrombocytopenia, elevated D-dimer and low fibrinogen levels may be seen as a consequence of coagulation activation and consumption of clotting factors [7]. Inflammatory markers such as $\mathrm{C}$ reactive protein (CRP) and erythrocyte sedimentation rate (ESR) are generally elevated; the alanine aminotransferase (ALT), aspartate aminotransferase (AST), prothrombin time, creatinine, Creatine phosphokinase (CPK), Lactate dehydrogenase (LDH) and ferritin amounts might increase by disease progress consequent to the general inflammation [56]. Radiologic findings for instance chest X-ray could be normal in the early stages of disease but show bilateral infiltrations in advanced stages. Computerized tomography $(\mathrm{CT})$ scan imaging is more sensitive and specific than simple X-ray and generally shows infiltrations, groundglass opacities and subsegmental consolidation in affected lung [29].

The most specified diagnostic tests for COVID-19 are molecular and serological laboratory tests. Obviously, the first step in molecular diagnosis is proper specimen collection. The nucleic acid amplification tests' specimen could be collected from the upper respiratory tract (nasopharyngeal and oropharyngeal swab or wash) or lower respiratory tract (sputum, endotracheal aspirate or bronchoalveolar lavage). SARS-CoV-2 RNA has also been detected in patients' blood and stool samples. In death cases it could be separated from lung tissue autopsies. Serological specimens are collected from patients' peripheral blood. The collected samples should be delivered to the laboratory at $2-8^{\circ} \mathrm{C}$. When there is likely to be a transfer delay the specimens must be frozen to $-20^{\circ} \mathrm{C}$ or ideally $-70^{\circ} \mathrm{C}$. It is important to avoid repeated freeze and thaw of samples. In addition, all specimens should be regarded as potentially infectious [47]. The most practical COVID-19 diagnostic tests are listed below.

\subsection{Nucleic acid amplification tests}

At present, the diagnosis of most COVID-19 suspicious cases is confirmed by reverse real-time transcriptionpolymerase chain reaction (rRT-PCR) test which detects the unique sequences of SARS-CoV-2 RNA [59]. Although RNA detection from respiratory samples (usually nasopharynx) is the standard for COVID-19 diagnosis, the sensitivity of testing varies with the time of sampling after exposure. One study showed the sensitivity of $33 \% 4$ days after exposure, $62 \%$ on the day of symptom onset, and $80 \% 3$ days after symptom onset [54]. In addition to the insufficient time post-exposure, inadequate specimen collection, different sources of sampling (bronchoalveolar lavage fluid with $93 \%$, sputum $72 \%$ nasal swabs $63 \%$, and pharyngeal swabs $32 \%$ of sensitivity) and technical difficulties are other factors contributing to the false negative results of RT-PCR tests [65].

\subsection{Serological testing}

Serological surveys could be used for studying the ongoing outbreak as well as retrospective assessment of an outbreak; however, the crossreactivity to the previous coronaviruses (e.g. common cold coronaviruses) and the time gap between exposure and antibody production may be challenging. Nonetheless, when rRT-PCR assays are negative and there is strong epidemiological evidence of SARS-CoV-2 infection, paired serum samples (in the acute and convalescent phase) could support the diagnosis [43]. Serological tests are faster and easier to perform and require less technical expertise and instrumental support compared to the nucleic acid detection tests; therefore, they could be used in basic clinical laboratories and smaller settings, reaching a wider application. At the moment, many commercial and noncommercial serological tests are under development and serological evaluation of infected and survived individuals is in progress, which will be discussed further below.

\subsection{Viral sequencing}

Genome sequencing of coronavirus is not only useful for confirming primary diagnosis by rRT-PCR, but also provides valuable information about eventual variations 
of virus. The results of SARS-CoV-2 sequencing are gathered in databases like GISAID being available for further studies [23]. Regarding technical complexities and expensive equipment required for genome sequencing it is not suitable for routine application.

\subsection{Viral culture}

SARS-CoV-2 culture could be performed using primary monkey cells and cell lines such as Vero; however, it is not recommended as a routine diagnostic procedure because it is time-consuming and requires biosafety level 2/3 laboratories [12,39]. A study on COVID-19 inpatients showed a high pharyngeal virus shedding during the first week of symptoms, with approximately 7 $\times 10^{8}$ RNA copies per throat swab on day 4 . The virus was isolated from throat or lung samples but not from stool (in spite of high concentrations of virus RNA), blood and urine samples. The shedding of viral RNA from sputum lasted until the end of symptoms. Seroconversion occurred after 7 days in $50 \%$ of patients (and by day 14 in all patients), but was not accompanied by decline in viral load. Viral nucleic acid could be detected in throat swabs for up to 6 weeks after disease onset whereas viral cultures were generally negative after 4 weeks [67].

\section{Treatment}

At the moment despite all efforts there is no definite medication to cure COVID-19; therefore, the patients receive supportive care such as supplemental oxygen therapy, anti-inflammatory drugs, broad-spectrum antibiotics (to cure or prevent from coinciding bacterial infections), anticoagulants (to prevent thromboembolic complications) and different antiviral agents [3]. Systemic corticosteroids (e.g. dexamethasone) are broadly used to control inflammatory response. Most antiviral drugs including neuraminidase inhibitors (oseltamivir, peramivir, zanamivir), ganciclovir, acyclovir, and ribavirin, which were commonly used to treat influenza infection has been shown to be invalid for COVID-19 and not recommended [16]. Nonetheless, among the RNA polymerase inhibitors including ribavirin, favipiravir, and remdesivir (GS-5734), the latter, a 1'-cyanosubstituted adenosine nucleotide analog that has broadspectrum antiviral activity against several RNA viruses has been reported to treat a number of COVID-19 cases successfully $[1,8,26]$. Chloroquine, the drug used for treating malaria has also shown some beneficial ef- fects possibly through suppressing the production and release of TNF- $\alpha$ and IL-6 cytokines [2]. Moreover, the protease inhibitors Lopinavir and Ritonavir used to treat human immunodeficiency virus (HIV) infection were able to decrease the viral loads of COVID-19 in patients [37]. Targeted immunomodulatory therapies for instance tocilizumab (anti-IL-6 mAb), sarilumab (anti-IL-6R mAb) and anakinra (IL-1 receptor antagonist) have been applied for managing cytokine storm in severe illness [4,19,20,44]. Tyrosine kinase inhibitors such as imatinib and ruxolitinib are also studied for their potential to prevent pulmonary vascular leakage [5,45]. Passive antibody therapy including infusion of convalescent plasma and IVIG are other options to treat severe cases $[11,70]$. Many clinicians have tried to apply a combination of abovementioned drugs to improve their efficacy [22]; however, there is not yet any declared consensus on a certain treatment protocol.

\section{Vaccine}

Currently, there is no human vaccine available for SARS-CoV-2, however, more than 120 candidates are under different phases of development including inactivated or live attenuated virus, viral vectors, recombinant proteins, virus particles and DNA or RNA vaccines. The main hurdles in producing an effective vaccine consist of technical barriers (e.g. selecting the most protective antigens, prior exposure to corona, need for adjuvant, feasibility of large scale production, viral mutations, duration of immunity, number of vaccination and intervals) and social challenges (e.g. human clinical trials, ensuring safety and effectiveness, technology transfer and licensure agreements) [42]. According to the studies, the SARS-CoV-2 S protein appears to be a promising immunogenic molecule to be addressed in designing future vaccines but whether targeting the fulllength protein or only the receptor-binding domain is sufficient to prevent transmission remains unclear [33].

\section{Diagnostic antibodies}

Serological tests are valuable particularly in epidemiologic studies and determining the immunity level of affected population including both diagnosed and asymptomatic individuals. Such studies might provide the primary platform for evaluation of tempting hypothesis like "Herd immunity". The high false-negative rate of nucleic acid amplification tests is also considered 
as a reason for developing supplementary diagnostic methods. In addition, as mentioned before, serological tests are more feasible in small laboratories with lesser facilities. In recent months, the researches have conducted several serological investigations using different techniques including enzyme-linked immunosorbent assay (ELISA) chemiluminescence immunoassay (CLIA), indirect immunofluorescence test (IIFT), lateral flow immunoassay, immunochromatography, western blot-based assays and virus neutralization assays. Among the 4 coronavirus structural proteins including spike (S), envelope (E), membrane (M), and nucleocapsid $(\mathrm{N}), \mathrm{S}$ and $\mathrm{N}$ proteins are the most studied antigens and almost all developing techniques concentrate on detecting the IgM and IgG antibodies produced against these molecules $[32,43]$.

Following SARS-CoV-2 infection, similar to the other infections, the titer of IgM raises first as an initial T-independent humoral response to the virus entry. Then antigen presentation to the $\mathrm{T}$ cells and isotype switching lead to the $\mathrm{IgG}$ production approximately within a week. According to the short half-life of IgM antibodies (5-6 days) and long half-life of $\operatorname{IgG}(21$ days) after a while, IgM amounts decrease to an undetectable level but IgG lasts for a longer period. Serological assays on COVID-19 patients demonstrate a similar pattern; for example, a retrospective study recruiting 112 patients with clinical symptoms of COVID19 infection evaluated IgM and IgG antibodies level against envelope protein (E) and nucleocapsid protein (N) with ELISA method. Among them 58 (51.79\%) patients were positive for both IgM and IgG, 7 (6.25\%) were negative for both antibodies, $1(0.89 \%)$ was positive for only $\operatorname{IgM}$, and $46(41.07 \%)$ were positive for only IgG. IgM antibodies appeared during the first week of disease onset, lasted for one month and gradually diminished; however, IgG antibodies were produced approximately 10 days after disease onset, and lasted longer [74]. Likewise, comparing the titer of $\mathrm{IgG}$ and IgM antibodies between two groups of 43 confirmed COVID-19 patients and 33 not-infected individuals using CLIA technique demonstrated greater diagnostic sensitivity for IgG than IgM (88.9\% vs $48.1 \%$ ) but fairly similar specificities $(90.9 \%$ vs $100 \%)$. Moreover, the IgM level increased and decreased over time but IgG positivity rate remained high for weeks [31]. Another study showed that both IgM and IgG titers were low or undetectable by ELISA at the first days of disease diagnosis but increased in almost all patients on day 5 as $\mathrm{IgM}$ and $\mathrm{IgG}$ positivity percent increased from $50 \%$ $(8 / 16)$ to $81 \%(13 / 16)$, and from $81 \%(13 / 16)$ to $100 \%$
(16/16) respectively [75]. Another group studying 173 patients with SARS-CoV-2 infection showed a seroconversion rate of $93.1 \%, 82.7 \%$ and $64.7 \%$ for total antibody, $\operatorname{IgM}$ and $\mathrm{IgG}$, while median seroconversion time for total antibody, IgM and IgG were day-11, day-12 and day-14, respectively. Moreover, less than $40 \%$ of patients had SARS-CoV-2antibodies in first week but it rapidly increased up to $100.0 \%$ for total $\mathrm{Ab}, 94.3 \%$ for IgM and $79.8 \%$ for IgG on day 15 . Notably, RNA detection rate by RT-PCR decreased from $66.7 \%$ in samples collected before day-7 to $45.5 \%$ during days 15-39. Therefore, it was suggested to use a combination of RNA and antibody detection methods to improve the sensitivity of COVID-19 diagnosis at early and late phases of disease. Furthermore, higher titers of total antibody were associated with worse prognosis [76] In another study on 16 patients with serum samples available 14 days or longer after symptom onset, rate of seropositivity was $94 \%$ for anti-N $\operatorname{IgG}(n=15)$, $88 \%$ for anti-N $\operatorname{IgM}(n=14), 100 \%$ for anti-RBD (receptor-binding domain) $\operatorname{IgG}(n=16)$, and $94 \%$ for anti-RBD IgM $(n=15)$. Moreover, the anti-SARSCoV-2-N and anti-SARS-CoV-2-RBD IgG levels were correlated with virus neutralization titer [62]. Evaluation of 208 plasma samples of 140 COVID-19 patients (confirmed and probable) by ELISA-based assay of the recombinant viral $\mathrm{N}$ protein showed that the median duration of $\operatorname{IgM}$ and $\operatorname{IgA}$ antibodies detection was 5 (interquartile range 3-6) days, while IgG was detectable 14 (IQR 10-18) days after disease onset, with a positive rate of $85.4 \%, 92.7 \%$, and $77.9 \%$, respectively. The sensitivity of IgM ELISA was higher than that of qPCR after 5 days of symptom onset and the positive detection rate improved significantly $(98.6 \%)$ by combination of IgM ELISA assay with qPCR test for each patient compared to a single qPCR test (51.9\%) [21]. Likewise, researchers studying the serum of 214 confirmed COVID19 patients using ELISA kits based on recombinant SARS-CoV-2 nucleocapsid protein (rN) and spike protein $(\mathrm{rS})$ reported $68.2 \%$ (146) and 70.1\% (150) of positivity with the rN-based IgM and IgG ELISAs, while $77.1 \%$ (165) and $74.3 \%$ (159) with the rS-based IgM and IgG ELISAs, respectively. The sensitivity of the rS-based ELISA for IgM detection was higher than that of the rN-based ELISA. The positive rate for IgM and IgG increased with disease duration particularly after 10 days, but the positive rate of $\operatorname{IgM}$ dropped on day 35 [38]. In addition, evaluation of the kinetics of IgM and IgA SARS-CoV-2 antibodies in 19 patients with confirmed (rRT-PCR) infection demonstrated that the IgM and IgA responses appear within 6-8 days of 
symptom onset. IgM-Ab levels peaked at 10-12 days and declined after 18 days whereas IgA-Ab peaks at 20-22 days, and is stronger and more persistent than the $\operatorname{IgM}$ response [49].

Regarding the urgent need for accurate, easy and rapid methods for screening the asymptomatic carriers in order to prevent virus transmission several companies and researches are making efforts to develop novel or modified techniques. One of these methods is lateral flow immunoassay which can detect total $\mathrm{Ab}, \mathrm{IgM}$ and/or IgG against SARS-CoV-2 antigens in blood, serum or plasma samples within a short time (e.g. 15 minutes). These kits are based on colloidal goldlabeled immunochromatography principle and one-step method using whole. A meta-analysis showed the sensitivity of these kits for both IgM and IgG tests between $72.7 \%$ and $100 \%$, while specificity ranged between $98.7 \%$ to $100 \%$ [53]. One group tested the validity of lateral flow immunoassay using samples collected from 397 PCR confirmed COVID-19 patients and 128 negative patients from different clinical sites and showed testing sensitivity and specificity of $88.66 \%$ and $90.63 \%$ for this method. In addition, they demonstrated detection consistency among samples from fingerstick blood, serum and plasma samples [36]. Other researchers applied the colloidal gold-based immunochromatographic (ICG) strip targeting anti-SARS-CoV-2 IgM or IgG antibody for serological assessment of COVID-19 infection. The sensitivity of ICG assay with IgM and IgG combinatorial detection in confirmed cases were $11.1 \%$, $92.9 \%$, and $96.8 \%$ at the early stage (first week), intermediate stage (second week), and late-stage (more two weeks after disease onset), respectively. The ICG detection capacity in nucleic acid-negative suspected cases was $43.6 \%$. In addition, the concordance of whole blood and plasma samples was reported to be acceptable [50].

The other technique used for evaluating the antibody responses to COVID-19 is magnetic chemiluminescence enzyme immunoassay (MCLIA) based on a double-antibody sandwich immunoassay. Using MCLIA it was shown that within 19 days after symptom onset in 285 patients, $100 \%$ of patients were positive for antiviral $\mathrm{IgG}$. Seroconversion for $\mathrm{IgG}$ and $\mathrm{IgM}$ occurred simultaneously or sequentially and both IgG and IgM titers plateaued within 6 days after seroconversion [40]. The other study applying CLIA technique (MAGLUMI 2000 Plus) investigated immunoglobulins kinetic in serum samples from COVID-19-positive patients at $<5$ days up until 26-30 days. The findings showed a rapid increase of both $\operatorname{IgM}$ and $\mathrm{IgG}$ after 67 days from the symptom onset. IgG had $100 \%$ sensi- tivity on day 12 whereas IgM showed $88 \%$ sensitivity at the same time [48].

Despite all novel methods which recently have been developed to facilitate the diagnosis of COVID-19, ELISA has been the most applied technique in serological studies. For instance, ELISA test based on the recombinant $\mathrm{N}$ protein of SARS-CoV-2 demonstrated the $\mathrm{IgM}$ and $\mathrm{IgG}$ seroconversion as early as the 4 th day after symptom onset in COVID-19 patients. In the confirmed patients sensitivity, specificity, positive predictive value (PPV), negative predictive value (NPV), and consistency rate of $\operatorname{IgM}$ were $77.3 \%$ (51/66), 100\%, 100\%, $80.0 \%$, and $88.1 \%$, and those of $\mathrm{IgG}$ were $83.3 .3 \%$ (55/66), 95.0\%, 94.8\%, 83.8\%, and $88.9 \%$. In patients with suspected COVID-19, sensitivity, specificity, PPV, NPV, and consistency rate of IgM were $87.5 \%$ (21/24), $100 \%, 100 \%, 95.2 \%$, and $96.4 \%$, and those of IgG were $70.8 \%$ (17/24), $96.6 \%, 85.0 \%, 89.1 \%$, and $88.1 \%$ [69]. In another study, IgM and IgG responses against SARSCoV-2 $\mathrm{N}$ and $\mathrm{S}$ protein have been evaluated after symptom onset in the intensive care unit (ICU) and non-ICU patients. Using ELISA technique for 130 blood samples it was found that N-IgM and S-IgM in some non-ICU patients reached a peak in the second week, while $\mathrm{N}$ $\mathrm{IgG}$ and $\mathrm{S}-\mathrm{IgG}$ continued to increase in the third week. The combined detection of $\mathrm{N}$ and S-specific IgM and IgG could identify up to the $75 \%$ of SARS-CoV-2 infected patients in the first week. S-IgG was significantly higher in non-ICU patients than in ICU patients in the third week. In contrast, N-IgG was significantly higher in ICU patients. Moreover, S-IgG elevation correlated with the decrease of CRP in non-ICU patients [58]. Furthermore, it has been shown that the critical cases had higher anti-RBD IgG than the mild/moderate cases but anti-RBD IgM OD were not correlated with disease severity. Additionally, confirmatory microneutralization (MN) and $90 \%$ plaque reduction neutralization tests (PRNT90) were positive after 4 weeks from disease onset in patients while there was no detectable crossreactivity in control group [51]. By retrospectively analyzing the data of viral RNAs and serum IgM-IgG antibodies against SARS-CoV-2 from 38 cases with confirmed COVID-19 it was found that at the early stages of the illness, the viral RNA is most abundant in the sputum specimens, followed by that in the throat swabs, while the antibody assays identify fewer positive cases at this stage. However, at the end of the first week of disease onset, the sensitivity of the serology assays outweighs the RNA test's. Notably most of cases with no detectable viral RNA load during the early stages were seropositive after 7 days. Therefore, simultaneous use 
of antibody assay and RT-PCR could improve the sensitivity of the diagnoses [73]. These findings indicate that serological tests could be used as efficient supplementary tools for detecting SARS-CoV-2 infection in suspected cases with no detectable viral RNA; furthermore, they could be useful in confirming the infection as well as to anticipate disease progression and prognosis as some studies showed that the higher titers of certain antibodies are accompanied by disease severity. Nonetheless, serological tests are not appropriate for early diagnosis because the least reported time length for IgM detection was 4 days after symptom onset while virus particles are detectable in respiratory tracts within the first days. Technical details should also be considered in interpretation of serological findings as antigen coating density, dilution matrix considerations, actual serum dilution and different cutoff point sets contribute to the various results obtained from serological evaluations. In addition, cross-reactivity with other strains of coronavirus, even other viruses, thus giving false positive results might affect serological tests accuracy.

\section{Therapeutic antibodies}

As mentioned before, due to the lack of specific medication to eliminate SARS-CoV-2, passive serotherapy is considered as an option for treating severe forms of COVID-19. This could be performed by producing monoclonal neutralizing antibodies or by plasma infusion from recovered individuals (convalescent plasma) to the critical patients. Previously, researchers had used the memory B cells of cured SARS-CoV patients for Epstein-Barr virus transformation and obtaining neutralizing antibodies [63]. Moreover, three human monoclonal antibodies (MAbs), m336, m337, and m338, targeting the receptor (CD26/DPP4) binding domain (RBD) of the MERS-CoV spike glycoprotein had been produced and suggested to be used for prophylactic and therapeutic purposes [71]. Regarding the considerable similarity between RBD of SARS-CoV and SARSCoV-2 it has recently been demonstrated that a SARSCoV-specific human monoclonal antibody, CR3022, could bind properly to the RBD of SARS-CoV-2; however, some of the most potent SARS-CoV-specific neutralizing antibodies (e.g.m396, CR3014) that target the ACE2 binding site of SARS-CoV failed to bind SARSCoV-2 S protein, implying that the difference in the RBD of SARS-CoV and SARS-CoV-2 has a critical impact on the cross-reactivity of neutralizing antibodies. Therefore, it is necessary to develop novel mono- clonal antibodies that could bind to the SARS-CoV-2 RBD [61].

Currently, the term "convalescent plasma (CP)" is used for describing the immune serum-containing antiSARS-CoV-2 antibodies which are considered to be helpful in treating critical COVID-19 cases. In a recent study, one dose of $200 \mathrm{ml}$ of CP derived from recovered donors with the neutralizing antibody titers above 1:640 was transfused beside antiviral drugs to 10 severe COVID-19 patients. The median time from disease onset to CP transfusion was 16 days. After CP transfusion, the level of neutralizing antibody increased rapidly up to 1:640 in five cases. The clinical symptoms were significantly improved oxyhemoglobin saturation increased, lymphocyte counts raised, CRP decreased and lung lesions were fairly resolved in radiological examinations; moreover, the viral load became undetectable in 7 patients. Notably, no severe adverse effect was observed [16]. In a similar study 5 critically ill COVID-19 patients with severe pneumonia and ARDS syndrome who had continuously high viral loads despite antiviral treatment with $\mathrm{PaO}_{2} / \mathrm{FiO}_{2}<300$, and under mechanical ventilation were treated with $\mathrm{CP}$ transfusion. Patients received $\mathrm{CP}$ with a SARS-CoV-2-specific IgG titer more than 1:1000 and a neutralization titer greater than 40 which had been collected from 5 recovered individuals. Convalescent plasma was administered between 10 and 22 days after admission. Following CP transfusion, body temperature normalized within 3 days in 4 of 5 patients and $\mathrm{PaO}_{2} / \mathrm{FiO}_{2}$ increased within 12 days. Viral load decreased and became negative within 12 days while SARS-CoV-2-specific neutralizing antibody titers increased, ARDS resolved in 4 patients at 12 days after transfusion, and 3 patients were weaned from mechanical ventilation. Three patients have been discharged from the hospital and 2 were in stable condition 37 days after transfusion [55].

According to the kinetic of SARS-CoV-2- IgM and $\mathrm{IgG}$ antibodies, there is an essential question about their protective potential against future reinfections. An old study in 1990 on 15 volunteers inoculated with coronavirus 229E had shown that although antibody concentrations were present one year later, this did not prevent reinfection when volunteers were challenged with the homologous virus; however, the period of virus shedding was shorter [10]. In an animal study, it was demonstrated that subsequent to intranasal administration of SARS-CoV to the mice, a neutralizing antibody response was induced which protected them from reinfection 4 weeks after primary infection. Moreover, passive transfer of immune serum to naïve mice pre- 
vented virus replication in the lower respiratory tract following intranasal challenge [57]. Similarly, a recent study on rhesus monkeys has shown that after primary infection with SARS-CoV-2, monkeys were protected against reinfection within four weeks after the first exposure as virus load remained comparable to the nonchallenged animals [6]; despite encouraging results of these studies, 4 weeks of follow up seems fairly insufficient to come to conclusion about antibodies protection; because as mentioned before, $\operatorname{IgM}$ titer remains high at least until 4 weeks and $\mathrm{IgG}$ is still near to its peak during this time. To clarify this point, it is suggested to conduct larger studied in longer time periods. One such study has been performed for SARS-CoV in which six years followed up of 23 SARS patients showed that SARS$\mathrm{CoV}$-specific IgG Abs were undetectable in most former patients. Although no SARS-CoV Ag-specific memory $\mathrm{B}$ cell response was detected, memory $\mathrm{T}$ cell responses to a pool of SARS-CoV S peptides were identified in 14 of $23(60.9 \%)$ of recovered individuals which was absent in close contact group. Patients with more severe clinical manifestations had more Ag-specific memory $\mathrm{T}$ cell [70].

The other question is about the cross-reactivity of neutralizing antibodies. It should be defined whether previous infections with a certain strain of corona virus could protect the individuals against future strains. Accordingly, a recent serological assessment of 175 COVID-19 recovered patients with mild symptoms using RBD, S1, and S2 antigens of SARS-CoV-2 demonstrated that SARS-CoV-2 neutralizing antibodies (NAbs) had no cross-reactivity with SARS-CoV virus. These antibodies were detectable in serum on day 1015 after disease onset and remained thereafter; however, their titers were variable in different patients. Elderly and middle-aged patients had significantly higher plasma neutralizing antibody titers than young patients. Moreover, there were ten patients with undetectable NAbs. The titers of neutralizing antibodies were positively correlated with plasma CRP levels and negatively correlated with the lymphocyte counts of patients at the time of admission [68]. In addition, a study reported that the mice inoculated with $S$ nanoparticles produced high-level neutralizing antibodies against homologous viruses, but these antibodies had no cross-protection against heteroviruses [9].

The other point to be defined in protective efficacy of anti-SARS-CoV-2 antibodies is the role of secretory IgAs in defense against reinfection. According to the fact that corona viruses infect and invade mainly the respiratory and gastrointestinal tracts, importance of studying the protective capacity of mucosal immunity becomes more clear. Previous studies on SARS-CoV had shown that intranasal vaccine challenges in mice would result in SIgA secretion in respiratory mucosa but whether this could inhibit viral invasion or not remains to be answered [15,41].

Another question to be discussed in passive serotherapy is "Antibody-Dependent Enhancement". It has yet been shown that patients with more severe clinical courses had earlier and higher antibody responses [24]. To explain this phenomenon, it is hypothesized that anti-S antibodies, while inhibiting viral entrance in permissive cells, potentiated the infection of B cells and macrophages by binding to their $\mathrm{Fc} \gamma$ receptor-II. Anti-S IgG antibodies bound to Fc $\gamma$ RII on mononuclear phagocyte cells promote viral entrance through phagocytosis thereby activating these cells and inducing proinflammatory cytokines release $[30,64,72]$. Regarding the probability of antibody-dependent enhancement, administration of CP or monoclonal antibodies have to be performed more cautiously because infusing high amount of antibodies might provoke inflammatory responses, exacerbating or triggering serious conditions like cytokine storm.

\section{Conclusion}

According to the urgent need of identifying the SARS-CoV-2-infected individuals in population researchers are trying to design rapid, sensitive specific and easy-to-do diagnostic tests with the least price. Besides, there are efforts to apply the natural potential of immune system for treating the COVID-19 patients. Anti-viral antibodies being suitable for both purposes are under diagnostic and therapeutic investigations. Among all studied viral antigens the spike (S) and nucleocapsid (N) proteins are considered as the most immunogenic antigens that antibodies against them could be detected in blood circulation of infected individuals making them suitable for serological testing. The principal advantages of serological tests are simplicity and the potential of detecting infected individuals after disease resolution thus providing the probability of conducting retrospective studies in great populations. Despite subtle disparities between the findings of different groups using various techniques, the overall results indicate that IgM antibodies appear within the first week of disease onset but wean after a month whereas IgG antibodies are produced during the second week and last for a long time (not exactly defined), moreover, 
there are very few studies about $\operatorname{IgA}$ antibodies which elevated during first weeks and were present in blood circulation longer than IgM. Secretory IgA antibodies due to their significance in mucosal immunity and vaccine efficacy should be studied more.

Neutralizing antibodies mainly targeting the receptor binding domain of S protein might be applied as supplementary option to treat severe forms of COVID-19 since administration of convalescent plasma containing sufficient titers of these antibodies to the critical patients has resulted in encouraging clinical improvements. Moreover, studying the neutralizing antibodies paves the way for finding the most appropriate antigenic targets to design efficient vaccines against SARS-CoV-2.

\section{Conflict of interest}

The authors declare no conflict of interest.

\section{References}

[1] M.L. Agostini, E.L. Andres, A.C. Sims, R.L. Graham, T.P. Sheahan, X. Lu, E.C. Smith, J.B. Case, J.Y. Feng and R. Jordan, Coronavirus susceptibility to the antiviral remdesivir (GS5734 ) is mediated by the viral polymerase and the proofreading exoribonuclease, MBio 9 (2018), e00221-00218.

[2] A.C. Aguiar, E. Murce, W.A. Cortopassi, A.S. Pimentel, M.M. Almeida, D.C. Barros, J.S. Guedes, M.R. Meneghetti and A.U. Krettli, Chloroquine analogs as antimalarial candidates with potent in vitro and in vivo activity, International Journal for Parasitology: Drugs and Drug Resistance 8 (2018), 459-464.

[3] W. Alhazzani, M.H. Møller, Y.M. Arabi, M. Loeb, M.N. Gong, E. Fan, S. Oczkowski, M.M. Levy, L. Derde and A.J.I.C.M. Dzierba, Surviving Sepsis Campaign: guidelines on the management of critically ill adults with Coronavirus Disease 2019 (COVID-19), 2020, 1-34.

[4] S.K. Alzghari and V.S.J.J.o.C.V. Acuña, Supportive treatment with tocilizumab for COVID-19: A systematic review, 127 (2020), 104380

[5] B.G. Bagca, C.B.J.C. Avci and G.F. Reviews, The Potential of JAK/STAT Pathway Inhibition by Ruxolitinib in the Treatment of COVID-19, S1359-6101, 1320, 30158-30151.

[6] L. Bao, W. Deng, H. Gao, C. Xiao, J. Liu, J. Xue, Q. Lv, J. Liu, P. Yu, Y. Xu, F. Qi, Y. Qu, F. Li, Z. Xiang, H. Yu, S. Gong, M. Liu, G. Wang, S. Wang, Z. Song, W. Zhao, Y. Han, L. Zhao, X. Liu, Q. Wei and C. Qin, Reinfection could not occur in SARS-CoV-2 infected rhesus macaques, bioRxiv, 2020, 2020.2003.2013.990226.

[7] R.C.J.J.o.T. Becker and Thrombolysis, COVID-19 update: Covid-19-associated coagulopathy, 2020, 1-14.

[8] J.H. Beigel, K.M. Tomashek, L.E. Dodd, A.K. Mehta, B.S. Zingman, A.C. Kalil, E. Hohmann, H.Y. Chu, A. Luetkemeyer and S.J.N.E.J.o.M. Kline, Remdesivir for the treatment of Covid-19 - preliminary report, 2020.

[9] D. Blanco-Melo, B. Nilsson-Payant, W.-C. Liu, R. Møller, M. Panis, D. Sachs and R. Albrecht, SARS-CoV-2 launches a unique transcriptional signature from in vitro, ex vivo, and in vivo systems, BioRxiv, 2020.
[10] K.A. Callow, H.F. Parry, M. Sergeant and D.A. Tyrrell, The time course of the immune response to experimental coronavirus infection of man, Epidemiology and Infection $\mathbf{1 0 5}$ (1990), 435-446.

[11] A. Casadevall and L.-a.J.T.J.o.c.i. Pirofski, The convalescent sera option for containing COVID-19, 130 (2020), 1545-1548.

[12] K.H. Chan, L.L. Poon, V. Cheng, Y. Guan, I. Hung, J. Kong, L.Y. Yam, W.H. Seto, K.Y. Yuen and J.S.M. Peiris, Detection of SARS coronavirus in patients with suspected SARS, Emerging Infectious Diseases 10 (2004), 294.

[13] M.A. Chowdhury, N. Hossain, M.A. Kashem, M.A. Shahid, A.J.J.o.I. Alam and P. Health, Immune Response in COVID19: A Review, 2020.

[14] V.J. Costela-Ruiz, R. Illescas-Montes, J.M. Puerta-Puerta, C. Ruiz, L.J.C. Melguizo-Rodríguez and G.F. Reviews, SARSCoV-2 infection: the role of cytokines in COVID-19 disease, 2020.

[15] L. Du, G. Zhao, Y. Lin, H. Sui, C. Chan, S. Ma, Y. He, S. Jiang, C. Wu, K.Y. Yuen, D.Y. Jin, Y. Zhou and B.J. Zheng, Intranasal vaccination of recombinant adeno-associated virus encoding receptor-binding domain of severe acute respiratory syndrome coronavirus (SARS-CoV) spike protein induces strong mucosal immune responses and provides long-term protection against SARS-CoV infection, J Immunol 180 (2008), 948-956.

[16] K. Duan, B. Liu, C. Li, H. Zhang, T. Yu, J. Qu, M. Zhou, L. Chen, S. Meng and Y. Hu, Effectiveness of convalescent plasma therapy in severe COVID-19 patients, Proceedings of the National Academy of Sciences 117 (2020), 9490-9496.

[17] R.L. Graham, E.F. Donaldson and R.S. Baric, A decade after SARS: Strategies for controlling emerging coronaviruses, Nature Reviews Microbiology 11 (2013), 836-848.

[18] L.E. Gralinski and V.D. Menachery, Return of the coronavirus: 2019-nCoV, Viruses 12 (2020), 135.

[19] G. Guaraldi, M. Meschiari, A. Cozzi-Lepri, J. Milic, R. Tonelli, M. Menozzi, E. Franceschini, G. Cuomo, G. Orlando and V.J.T.L.R. Borghi, Tocilizumab in patients with severe COVID19: a retrospective cohort study, 2020.

[20] E. Gubernatorova, E. Gorshkova, A. Polinova, M.J.C. Drutskaya and G.F. Reviews, IL-6: relevance for immunopathology of SARS-CoV-2, 2020.

[21] L. Guo, L. Ren, S. Yang, M. Xiao, D. Chang, F. Yang, C.S. Dela Cruz, Y. Wang, C. Wu, Y. Xiao, L. Zhang, L. Han, S. Dang, Y. Xu, Q.-W. Yang, S.-Y. Xu, H.-D. Zhu, Y.-C. Xu, Q. Jin, L. Sharma, L. Wang and J. Wang, Profiling Early Humoral Response to Diagnose Novel Coronavirus Disease (COVID19), Clinical Infectious Diseases, 2020.

[22] Y.-R. Guo, Q.-D. Cao, Z.-S. Hong, Y.-Y. Tan, S.-D. Chen, H.-J. Jin, K.-S. Tan, D.-Y. Wang and Y. Yan, The origin, transmission and clinical therapies on coronavirus disease 2019 (COVID-19) outbreak - an update on the status, Military Medical Research 7 (2020), 1-10.

[23] D.L. Heymann and N. Shindo, COVID-19: What is next for public health? The Lancet 395 (2020), 542-545.

[24] M.-S. Ho, W.-J. Chen, H.-Y. Chen, S.-F. Lin, M.-C. Wang, J. Di, Y.-T. Lu, C.-L. Liu, S.-C. Chang, C.-L. Chao, C.-C. King, J.-M. Chiou, I.-J. Su and J.-Y. Yang, Neutralizing antibody response and SARS severity, Emerging Infectious Diseases 11 (2005), 1730-1737.

[25] M. Hoffmann, H. Kleine-Weber, S. Schroeder, N. Krüger, T. Herrler, S. Erichsen, T.S. Schiergens, G. Herrler, N.-H. Wu, A. Nitsche, M.A. Müller, C. Drosten and S. Pöhlmann, SARSCoV-2 cell entry depends on ACE2 and TMPRSS2 and is blocked by a clinically proven protease inhibitor, Cell $\mathbf{1 8 1}$ (2020), 271-280.e278. 
[26] M.L. Holshue, C. DeBolt, S. Lindquist, K.H. Lofy, J. Wiesman, H. Bruce, C. Spitters, K. Ericson, S. Wilkerson and A. Tural, First case of 2019 novel coronavirus in the United States, New England Journal of Medicine, 2020.

[27] C. Huang, Y. Wang, X. Li, L. Ren, J. Zhao, Y. Hu, L. Zhang, G. Fan, J. Xu, X. Gu, Z. Cheng, T. Yu, J. Xia, Y. Wei, W. Wu, X. Xie, W. Yin, H. Li, M. Liu, Y. Xiao, H. Gao, L. Guo, J. Xie, G. Wang, R. Jiang, Z. Gao, Q. Jin, J. Wang and B. Cao, Clinical features of patients infected with 2019 novel coronavirus in Wuhan, China, Lancet 395 (2020), 497-506.

[28] I. Huang and R. Pranata, Lymphopenia in severe coronavirus disease-2019 (COVID-19): Systematic review and metaanalysis, Journal of Intensive Care $\mathbf{8}$ (2020), 36.

[29] P. Huang, T. Liu, L. Huang, H. Liu, M. Lei, W. Xu, X. Hu, J. Chen and B. Liu, Use of chest CT in combination with negative RT-PCR assay for the 2019 novel coronavirus but high clinical suspicion, Radiology 295 (2020), 22-23.

[30] M. Jaume, M.S. Yip, C.Y. Cheung, H.L. Leung, P.H. Li, F Kien, I. Dutry, B. Callendret, N. Escriou, R. Altmeyer, B. Nal, M. Daëron, R. Bruzzone and J.S.M. Peiris, Anti-severe acute respiratory syndrome coronavirus spike antibodies trigger infection of human immune cells via a $\mathrm{pH}$ - and cysteine protease-independent $\mathrm{Fc} \gamma \mathrm{R}$ pathway, Journal of Virology 85 (2011), 10582-10597.

[31] Y. Jin, M. Wang, Z. Zuo, C. Fan, F. Ye, Z. Cai, Y. Wang, H. Cui, K. Pan and A. Xu, Diagnostic value and dynamic variance of serum antibody in coronavirus disease 2019, International Journal of Infectious Diseases, 2020.

[32] A. La Marca, M. Capuzzo, T. Paglia, L. Roli, T. Trenti and S.M.J.R.b.o. Nelson, Testing for SARS-CoV-2 (COVID-19): systematic review and clinical guide to molecular and serological in-vitro diagnostic assays, 2020

[33] T.T. Le, Z. Andreadakis, A. Kumar, R.G. Roman, S. Tollefsen, M. Saville and S.J.N.R.D.D. Mayhew, The COVID-19 vaccine development landscape, 19 (2020), 305-306.

[34] G. Li, Y. Fan, Y. Lai, T. Han, Z. Li, P. Zhou, P. Pan, W. Wang, D. $\mathrm{Hu}$ and $\mathrm{X}$. Liu, Coronavirus infections and immune responses, Journal of Medical Virology 92 (2020), 424-432.

[35] Y. Li, M. Chen, H. Cao, Y. Zhu, J. Zheng, H.J.M. Zhou and infection, Extraordinary GU-rich single-strand RNA identified from SARS coronavirus contributes an excessive innate immune response, 15 (2013), 88-95.

[36] Z. Li, Y. Yi, X. Luo, N. Xiong, Y. Liu, S. Li, R. Sun, Y. Wang, B. Hu, W. Chen, Y. Zhang, J. Wang, B. Huang, Y. Lin, J. Yang, W. Cai, X. Wang, J. Cheng, Z. Chen, K. Sun, W. Pan, Z. Zhan, L. Chen and F. Ye, Development and clinical application of a rapid IgM-IgG combined antibody test for SARS-CoV-2 infection diagnosis, J Med Virol, 2020.

[37] J. Lim, S. Jeon, H.-Y. Shin, M.J. Kim, Y.M. Seong, W.J. Lee, K.-W. Choe, Y.M. Kang, B. Lee and S.-J. Park, Case of the index patient who caused tertiary transmission of COVID-19 infection in Korea: The application of lopinavir/ritonavir for the treatment of COVID-19 infected pneumonia monitored by quantitative RT-PCR, Journal of Korean Medical Science $\mathbf{3 5}$ (2020).

[38] W. Liu, L. Liu, G. Kou, Y. Zheng, Y. Ding, W. Ni, Q. Wang, L. Tan, W. Wu, S. Tang, Z. Xiong and S. Zheng, Evaluation of Nucleocapsid and Spike Protein-based ELISAs for detecting antibodies against SARS-CoV-2, J Clin Microbiol, 2020.

[39] M.J. Loeffelholz and Y.-W. Tang, Laboratory diagnosis of emerging human coronavirus infections - the state of the art, Emerging microbes \& infections, 2020, 1-26.

[40] Q.X. Long, B.Z. Liu, H.J. Deng, G.C. Wu, K. Deng, Y.K. Chen, P. Liao, J.F. Qiu, Y. Lin, X.F. Cai, D.Q. Wang, Y. Hu,
J.H. Ren, N. Tang, Y.Y. Xu, L.H. Yu, Z. Mo, F. Gong, X.L. Zhang, W.G. Tian, L. Hu, X.X. Zhang, J.L. Xiang, H.X. Du, H.W. Liu, C.H. Lang, X.H. Luo, S.B. Wu, X.P. Cui, Z. Zhou, M.M. Zhu, J. Wang, C.J. Xue, X.F. Li, L. Wang, Z.J. Li, K. Wang, C.C. Niu, Q.J. Yang, X.J. Tang, Y. Zhang, X.M. Liu, J.J. Li, D.C. Zhang, F. Zhang, P. Liu, J. Yuan, Q. Li, J.L. Hu, J. Chen and A.L. Huang, Antibody responses to SARS-CoV-2 in patients with COVID-19, Nat Med, 2020.

[41] B. Lu, Y. Huang, L. Huang, B. Li, Z. Zheng, Z. Chen, J. Chen, Q. Hu and H. Wang, Effect of mucosal and systemic immunization with virus-like particles of severe acute respiratory syndrome coronavirus in mice, Immunology 130 (2010), 254261

[42] N. Lurie, M. Saville, R. Hatchett and J.J.N.E.J.o.M. Halton, Developing Covid-19 vaccines at pandemic speed, 382 (2020), 1969-1973.

[43] B. Meyer, C. Drosten and M.A. Müller, Serological assays for emerging coronaviruses: Challenges and pitfalls, Virus Research 194 (2014), 175-183.

[44] L.A. Monteagudo, A. Boothby and E.J.A.o.r. Gertner, Continuous intravenous anakinra infusion to calm the cytokine storm in macrophage activation syndrome, 2 (2020), 276-282.

[45] A. Morales-Ortega, D. Bernal-Bello, C. Llarena-Barroso, B Frutos-Pérez, M.Á. Duarte-Millán, V.G. de Viedma-García, A.I. Farfán-Sedano, E. Canalejo-Castrillero, J.M. Ruiz-Giardin and J.J.C.I. Ruiz-Ruiz, Imatinib for COVID-19: A case report, 2020 .

[46] T. Nelemans and M. Kikkert, Viral innate immune evasion and the pathogenesis of emerging RNA virus infections, Viruses 11 (2019), 961.

[47] W.H. Organization, Laboratory testing for coronavirus disease 2019 (COVID-19) in suspected human cases: interim guidance, 2 March 2020, in, World Health Organization, 2020.

[48] A. Padoan, C. Cosma, L. Sciacovelli, D. Faggian and M. Plebani, Analytical performances of a chemiluminescence immunoassay for SARS-CoV-2 IgM/IgG and antibody kinetics, Clin Chem Lab Med, 2020.

[49] A. Padoan, L. Sciacovelli, D. Basso, D. Negrini, S. Zuin, C. Cosma, D. Faggian, P. Matricardi and M. Plebani, IgA-Ab response to spike glycoprotein of SARS-CoV-2 in patients with COVID-19: A longitudinal study, Clin Chim Acta 507 (2020), 164-166.

[50] Y. Pan, X. Li, G. Yang, J. Fan, Y. Tang, J. Zhao, X. Long, S. Guo, Z. Zhao, Y. Liu, H. Hu, H. Xue and Y. Li, Serological immunochromatographic approach in diagnosis with SARSCoV-2 infected COVID-19 patients, J Infect, 2020.

[51] R.A. Perera, C.K. Mok, O.T. Tsang, H. Lv, R.L. Ko, N.C. Wu, M. Yuan, W.S. Leung, J.M. Chan, T.S. Chik, C.Y. Choi, K. Leung, K.H. Chan, K.C. Chan, K.C. Li, J.T. Wu, I.A. Wilson, A.S. Monto, L.L. Poon and M. Peiris, Serological assays for severe acute respiratory syndrome coronavirus 2 (SARS-CoV2), March 2020, Euro Surveill 25 (2020).

[52] D. Pinto, Y.-J. Park, M. Beltramello, A.C. Walls, M.A. Tortorici, S. Bianchi, S. Jaconi, K. Culap, F. Zatta and A.J.N. De Marco, Cross-neutralization of SARS-CoV-2 by a human monoclonal SARS-CoV antibody, 2020, 1-6.

[53] Z.Z. Rashid, S.N. Othman, M.N.A. Samat, U.K. Ali and K.K.J.T.M.J.o.P. Wong, Diagnostic performance of COVID-19 serology assays, 42 (2020), 13-21.

[54] N. Sethuraman, S.S. Jeremiah and A. Ryo, Interpreting diagnostic tests for SARS-CoV-2, Jama 323 (2020), 2249-2251.

[55] C. Shen, Z. Wang, F. Zhao, Y. Yang, J. Li, J. Yuan, F. Wang, D. Li, M. Yang, L. Xing, J. Wei, H. Xiao, Y. Yang, J. Qu, L. Qing, L. Chen, Z. Xu, L. Peng, Y. Li, H. Zheng, F. Chen, K. Huang, 
Y. Jiang, D. Liu, Z. Zhang, Y. Liu and L. Liu, Treatment of 5 Critically Ill Patients With COVID-19 With Convalescent Plasma, Jama, 2020.

[56] T. Singhal, A review of coronavirus disease-2019 (COVID-19), The Indian Journal of Pediatrics, 2020, 1-6.

[57] K. Subbarao, J. McAuliffe, L. Vogel, G. Fahle, S. Fischer, K. Tatti, M. Packard, W.-J. Shieh, S. Zaki and B. Murphy, Prior infection and passive transfer of neutralizing antibody prevent replication of severe acute respiratory syndrome coronavirus in the respiratory tract of mice, Journal of Virology 78 (2004), 3572-3577.

[58] B. Sun, Y. Feng, X. Mo, P. Zheng, Q. Wang, P. Li, P. Peng, X. Liu, Z. Chen, H. Huang, F. Zhang, W. Luo, X. Niu, P. Hu, L. Wang, H. Peng, Z. Huang, L. Feng, F. Li, F. Zhang, F. Li, N. Zhong and L. Chen, Kinetics of SARS-CoV-2 specific IgM and IgG responses in COVID-19 patients, Emerg Microbes Infect, 2020, 1-36.

[59] A. Tahamtan and A. Ardebili, Real-time RT-PCR in COVID19 detection: Issues affecting the results, Expert Review of Molecular Diagnostics 20 (2020), 453-454.

[60] W. Tai, L. He, X. Zhang, J. Pu, D. Voronin, S. Jiang, Y. Zhou and L. Du, Characterization of the receptor-binding domain (RBD) of 2019 novel coronavirus: implication for developmen of RBD protein as a viral attachment inhibitor and vaccine, Cellular \& Molecular Immunology, 2020.

[61] X. Tian, C. Li, A. Huang, S. Xia, S. Lu, Z. Shi, L. Lu, S. Jiang, Z. Yang and Y. Wu, Potent binding of 2019 novel coronavirus spike protein by a SARS coronavirus-specific human monoclonal antibody, Emerging Microbes \& Infections 9 (2020), 382-385.

[62] K.K. To, O.T. Tsang, W.S. Leung, A.R. Tam, T.C. Wu, D.C. Lung, C.C. Yip, J.P. Cai, J.M. Chan, T.S. Chik, D.P. Lau, C.Y Choi, L.L. Chen, W.M. Chan, K.H. Chan, J.D. Ip, A.C. Ng, R.W. Poon, C.T. Luo, V.C. Cheng, J.F. Chan, I.F. Hung, Z. Chen, H. Chen and K.Y. Yuen, Temporal profiles of viral load in posterior oropharyngeal saliva samples and serum antibody responses during infection by SARS-CoV-2: an observational cohort study, Lancet Infect Dis, 2020.

[63] E. Traggiai, S. Becker, K. Subbarao, L. Kolesnikova, Y. Uematsu, M.R. Gismondo, B.R. Murphy, R. Rappuoli and A. Lanzavecchia, An efficient method to make human monoclonal antibodies from memory B cells: Potent neutralization of SARS coronavirus, Nature Medicine 10 (2004), 871-875.

[64] Y. Wan, J. Shang, S. Sun, W. Tai, J. Chen, Q. Geng, L. He, Y. Chen, J. Wu and Z.J.J.o.v. Shi, Molecular mechanism for antibody-dependent enhancement of coronavirus entry, 94 (2020).

[65] W. Wang, Y. Xu, R. Gao, R. Lu, K. Han, G. Wu and W. Tan, Detection of SARS-CoV-2 in different types of clinical specimens, Jama 323 (2020), 1843-1844.
[66] W.J. Wiersinga, A. Rhodes, A.C. Cheng, S.J. Peacock and H.C.J.J. Prescott, Pathophysiology, Transmission, Diagnosis, and Treatment of Coronavirus Disease 2019 (COVID-19): A Review.

[67] R. Wölfel, V.M. Corman, W. Guggemos, M. Seilmaier, S. Zange, M.A. Müller, D. Niemeyer, T.C. Jones, P. Vollmar and C.J.N. Rothe, Virological assessment of hospitalized patients with COVID-2019, 581 (2020), 465-469.

[68] F. Wu, A. Wang, M. Liu, Q. Wang, J. Chen, S. Xia, Y. Ling, Y. Zhang, J. Xun and L. Lu, Neutralizing antibody responses to SARS-CoV-2 in a COVID-19 recovered patient cohort and their implications, 2020.

[69] F. Xiang, X. Wang, X. He, Z. Peng, B. Yang, J. Zhang, Q Zhou, H. Ye, Y. Ma, H. Li, X. Wei, P. Cai and W.L. Ma, Antibody Detection and Dynamic Characteristics in Patients with COVID-19, Clin Infect Dis, 2020.

[70] Y. Xie, S. Cao, H. Dong, Q. Li, E. Chen, W. Zhang, L. Yang, S. Fu and R.J.J.o.I. Wang, Effect of regular intravenous immunoglobulin therapy on prognosis of severe pneumonia in patients with COVID-19, 2020.

[71] T. Ying, L. Du, T.W. Ju, P. Prabakaran, C.C. Lau, L. Lu, Q Liu, L. Wang, Y. Feng and Y. Wang, Exceptionally potent neutralization of middle east respiratory syndrome coronavirus by human monoclonal antibodies, Journal of Virology 88 (2014), 7796-7805.

[72] M.S. Yip, N.H.L. Leung, C.Y. Cheung, P.H. Li, H.H.Y. Lee, M Daëron, J.S.M. Peiris, R. Bruzzone and M. Jaume, Antibodydependent infection of human macrophages by severe acute respiratory syndrome coronavirus, Virology Journal 11 (2014) 82.

[73] G. Yong, Y. Yi, L. Tuantuan, W. Xiaowu, L. Xiuyong, L. Ang and $\mathrm{H}$. Mingfeng, Evaluation of the auxiliary diagnostic value of antibody assays for the detection of novel coronavirus (SARS-CoV-2), J Med Virol, 2020.

[74] G. Zhang, S. Nie, Z. Zhang and Z. Zhang, Longitudinal Change of SARS-Cov2 Antibodies in Patients with COVID-19, J Infect Dis, 2020.

[75] W. Zhang, R.-H. Du, B. Li, X.-S. Zheng, X.-L. Yang, B. Hu Y.-Y. Wang, G.-F. Xiao, B. Yan and Z.-L. Shi, Molecular and serological investigation of 2019-nCoV infected patients: Implication of multiple shedding routes, Emerging Microbes \& Infections 9 (2020), 386-389.

[76] J. Zhao, Q. Yuan, H. Wang, W. Liu, X. Liao, Y. Su, X. Wang, J. Yuan, T. Li, J. Li, S. Qian, C. Hong, F. Wang, Y. Liu, Z. Wang, Q. He, Z. Li, B. He, T. Zhang, Y. Fu, S. Ge, L. Liu, J. Zhang, N. Xia and Z. Zhang, Antibody responses to SARS-CoV-2 in patients of novel coronavirus disease 2019, Clin Infect Dis, 2020. 